\title{
Prosthodontic management of surgically treated oral sub mucous fibrosis using the oral screen prosthesis
}

\author{
Dr. Chetan Oswal ${ }^{1}$, Dr. Paresh Gandhi ${ }^{2}$, Dr. Ajay Sabane ${ }^{3}$ \\ Post Graduate Student ${ }^{1}$, Professor ${ }^{2}$, Reader ${ }^{3}$ \\ Department of Prosthodontics, Bharati Vidyapeeth Dental College \& Hospital, Pune 411037, Maharashtra, \\ India
}

\begin{abstract}
OSMF is a chronic, progressive precancerous condition of oral mucosa, predominantly seen in the Indian subcontinent. A progressive inability to open the mouth fully, is an important feature in oral sub mucous fibrous due to the formation of fibrous bands especially in the buccal mucosa. In extreme cases, surgical excision of the fibrotic bands is the only treatment modality, but relapse is the major problem. This article describes the use of oral screen prosthesis used as an adjunct to surgery to prevent relapse.
\end{abstract}

Key Words: Oral sub mucous fibrosis, Nasolabial Flap, Oral Screen.

\section{Introduction}

Oral sub mucous fibrosis is an insidious, chronic, disabling disease of obscure etiology that affects the entire oral cavity, sometimes the pharynx, and rarely the larynx. It is characterized by blanching and stiffness of the oral mucosa, which causes progressive limitation of mouth opening and intolerance to hot and spicy food.

It is an established precancerous condition with increased prevalence in the Indian subcontinent. Its precancerous nature was first described by Paymaster, who recorded the onset of slowly growing squamous cell carcinomas in one third of patients with it. Minti et al reported a malignant transformation rate of $7.6 \%$.

The WHO definition for an oral precancerous condition - a generalized pathological state of the oral mucosa associated with a significantly increased risk of cancer.

The prodromal symptoms includes a burning sensation in the mouth when consuming spicy food, appearance of blisters especially on the palate, ulcerations or recurrent generalized inflammation of the oral mucosa, excessive salivation, defective gustatory sensation and dryness of mouth. As the disease progress, the oral mucosa becomes blanched and slightly opaque, and white fibrous bands appear. The buccal mucosa and lips may be affected at an early stage. The oral mucosa is involved symmetrically and the fibrous band in the buccal mucosa runs in a vertical direction. With progressing fibrosis, the stiffening of mucosa leads to difficulty in opening the mouth, inability to whistle or blow and difficulty in swallowing.

Treatments for oral sub mucous fibrosis are mainly symptomatic, because the etiology of the disease is not fully understood and it is progressive. Conservative treatment includes vitamins, iron supplements; intralesional injections of hyaluronidase, placental extracts, and steroids. In severe situations (interincisal opening less than or equal to $25 \mathrm{~mm}$ ), surgical intervention is the only treatment option. Surgical methods reported in the literature include various grafting procedures such as the split thickness skin graft, island palatal graft, bilateral tongue flaps, buccal fat pad graft, free forearm flap transfer, and bilateral full thickness nasolabial flaps.

Adequate stabilizationof the graft at the recipient site is essential for its unhindered acceptance and uneventful healing. Failure to secure the graft in position initially may result in rejection of the graft, subsequent relapse of the corrected mouth opening, and recurrence of the condition. Huang et al advocated use of custommade aluminous eye patches secured with transcutaneous sutures to stabilize the graft. Le and Gornitsky used an oral stent for 6 months to prevent relapse of the corrected mouth opening. Patil et al fabricated and used a graft stabilizing clip (GSC) to ensure positive contact of the graft at the recipient site.

This article describes the fabrication and use of oral screen prosthesis in such situations. The prosthesis helps to stabilize the secured flaps to and prevent it from being bitten into occlusion. It is simple in design, easy to fabricate, and similar to the orthodontic oral screen appliance used in patients with mouth breathers. The appliance can also be used as an oral stent to prevent relapse. It can be worn, removed, and cleaned by the patient easily. Being removable, it facilitates periodic examination of the surgical site.

\section{Case Report}

A 19 year old male patient came with a complaint of increasing difficulty of mouth opening and mastication for the previous 3 years. The patient had a habit of chewing betel nuts (along with tobacco and other unspecified ingredients) four to five times a day for 6 to 8 years. He would keep the mixture in the mouth, again the cheek (usually the right side) for approximately 20 minutes each time, chew, and finally spit it out. At the 
time of his first visit to Bharati hospital, he had a 2 year history of treatment in three hospitals, which had always resulted in relapse and worsening of the signs and symptoms.

The patient was seen with severely restricted mouth opening (approximately $15 \mathrm{~mm}$ ), (Fig. 1), which compromised his ability to eat and speak. Characteristic intra oral signs of OSF were seen including burning sensation on consuming spicy food blanched fibrotic tissue on the right and left buccal mucosa. A biopsy confirmed sub mucous fibrosis. Surgical intervention was carried out on patient's consent to improve his mouth opening.

The patient was operated under general anesthesia with nasal intubation. The trismus was released, buccal fibrotic tissues were excised, and nasolabial flap was reflected and secured over the defect with simple interrupted sutures (Fig. 2). The fabrication of appliance was made after 2 days.

\section{Ii.1 Fabrication Of The Appliance:}

An irreversible hydrocolloid impression (Zhermack, Italy), was recorded of both the upper and lower arches using stock metal trays. (Fig. 3) The impressions were poured using type III dental stone (Kalabhai, Mumbai). The casts were then hand articulated and sealed with modelling wax from lingual side (Fig. 4). A 20 $\mathrm{cm}$ long piece of 19 guage stainless steel orthodontic wire was then manipulated along the arch with a coil at the center (1 cm in diameter) to hold the appliance. (Fig. 5)

A two layer of modelling wax was adapted along the articulated casts incorporating the wire. The whole wax up was then processed in heat polymerizing acrylic resin using convectional techniques. It was then finished and polished in the usual manner. Holes were made in the anterior region for the ease of breathing. (Fig. 6)

The appliance was then inserted in the patient's mouth positioning it in the labial and buccal vestibule as oral screen. The appliance was adjusted according to patient's convenience (Fig. 7). Care was taken to avoid excess pressure, which might result in necrosis and rejection of the flap. The patient was educated regarding the proper insertion and removal and maintenance of the oral screen appliance.

The patient was encouraged to wear the appliance for 24 hours per day initially, for 1 week unless discomfort or injury occurs. After 1 week, the patient was told to use the appliance during the day time, and to remove at bed time for a month. The oral screen appliance was intended to use as an oral stent along with mouth exercises to prevent relapse of the corrected mouth opening. The patient was scheduled for monthly recall visits for 6 months or longer.

\section{Discussion}

Oral sub mucous fibrosis (OSMF) is an insidious chronic disabling disease involving oral mucosa, oropharynx and rarely larynx characterized by juxta epithelial inflammatory reaction followed by progressive fibrosis of the lamina propria and deeper connective tissues with concomitant muscle degeneration. The cause of OSMF is believed to be multifactorial a like betel nut chewing, areca nut chewing, ingestion of chillies, genetic and immunologic processes, nutritional deficiencies, and others. As the pathogenesis of the disease is not well established, its treatment has largely been symptomatic. Various treatment modalities including removing the etiology, mouth opening exercises, steroid injections and various surgical modalities have been described in literature for treating OSMF.

Surgical treatment is required in all advanced cases. The aim of surgical treatment is to release fibrotic bands and provide adequate mouth opening. Excision of the fibrous bands and propping the mouth open to allow secondary epithelialisation causes rebound fibrosis during healing.

The use of an oral screen as a treatment adjunct to surgery should be considered especially when the surgical technique is prone to relapse. The patient had to wear the oral screen for a prolonged period of time (6 months in this case) once the jaws have been stretched to allow the tissue to heal at the new, increased opening position. Because OSMF is progressive in nature and is by no means cured after the surgery, the patient was instructed to continue wearing the oral appliance weekly and perform the jaw stretching exercises daily.

\section{Conclusion}

This clinical case describes the prosthodontic approach to treat surgical correction of oral sub mucous fibrosis. Surgical correction involves excision of fibrotic bands and securing it with split thickness grafts to repair the resultant defect. But the possibility of graft shrinkage and interference with occlusion results in surgical failure of the treatment. Securing the flap in position using oral screen prosthesis has been tried as an adjunct to treat such cases. Hence, this modified approach can be performed in routine clinical practice, making the rehabilitation of such a case possible with relative ease. 


\section{References}

[1] Shafer WG. A textbook of oral pathology.4th ed. Philadelphia: WB Saunders Co; 1983. p. 86-229.

[2] Huang IY, Wu CF, Shen YS, Yang CF, ShiehTY, Hsu HJ, et al. Importance of patient's cooperation in surgical treatment for oral sub mucous fibrosis. J Oral MaxillofacSurg2008; 66:699-703.

[3] Morawetz G, Katsikeris N, Weinberg S, Listrom R. Oral sub mucous fibrosis. Int J OralMaxillofacSurg 1987; 16:609-14

[4] Mukherjee AL.Oralsubmucous Fibrosis. A search for aetiology.Ind J Otolaryngol 1972; 24:1:11-15.

[5] Borle RM, Borle SR. Management of oral sub mucous fibrosis: a conservative approach. J Oral MaxillofacSurg 1991; 49:788-791.

[6] Khanna JN, Andrade NN. Oral sub mucous fibrosis: a new concept in surgical management. Report of 100 cases. Int J Oral MaxillofacSurg 1995; 24:433-9.

[7] Yeh CJ. Application of the buccal fat pad to the surgical treatment of the oral sub mucous fibrosis. Int J oral MaxillofacSurg 1996; 25:130-3.

[8] Pravinkumar G. Patil, Rambhau D. Parkhedkar. New graft-stabilizing clip as a treatment adjunct for oral sub mucous fibrosis. J Prosthet Dent. 2009; 102:191-192.

[9] Kavarana NM, Bhathena HM. Surgery forsevere trismus in sub mucous fibrosis. Br J Plast Surg 1987; 40:407-9.

[10] Cunha-Gomes D, Kavarana NM, Choudhari C, Rajendraprasad JS, Bhathena HM, Desai PB, et al. Total oral reconstruction for cancers associated with advanced oral sub mucous fibrosis. Ann PlastSurg2003; 51:283-9.

[11] Le PV, Gornitsky M, Domanowski G. Oral stent as treatment adjunct for oral submucousfibrosis. Oral Surg Oral Med Oral Pathol Oral RadiolEndod 1996;81:148-50.

[12] Thomas KF. Prosthetic rehabilitation. Chicago: Quintessence; 1994. p. 5560.

[13] Morrow RM, Rudd KD, Rhoads JE. Dental laboratory procedures: complete dentures, Vol 1. 2nd ed. St. Louis: Mosby; 1986. p 312-38.

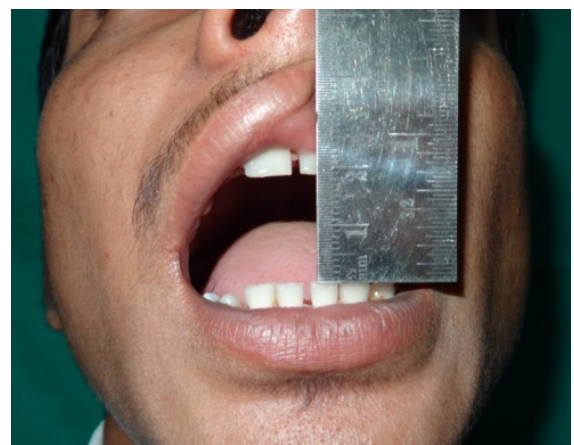

Fig: 1 Restricted mouth opening
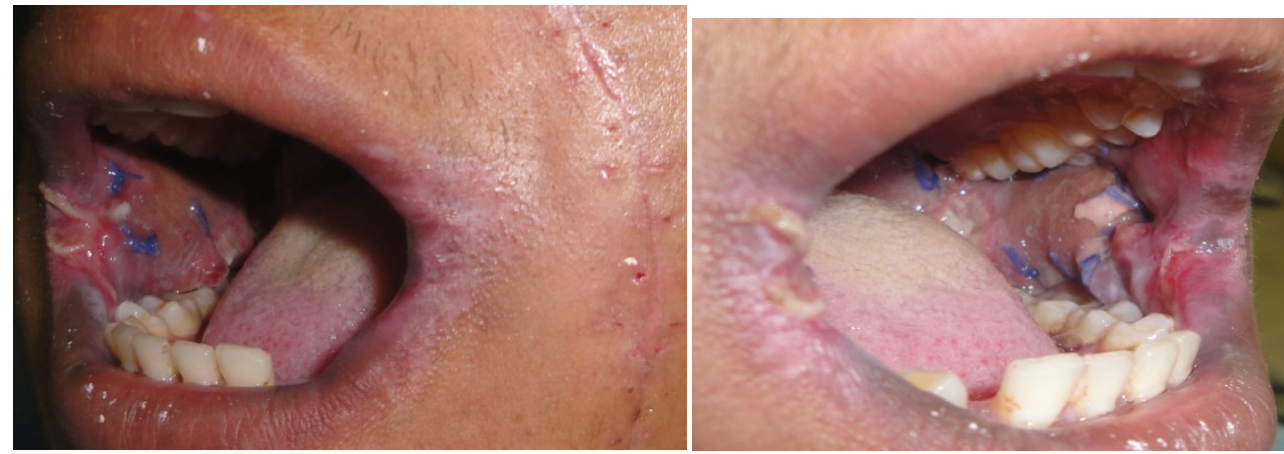

Fig: 2 Nasolabial flap secured with interrupted sutures.

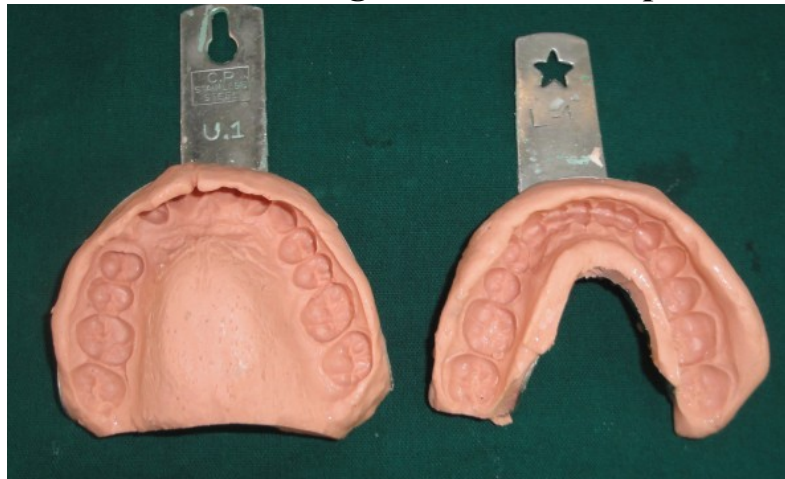

Fig: 3 Impressions

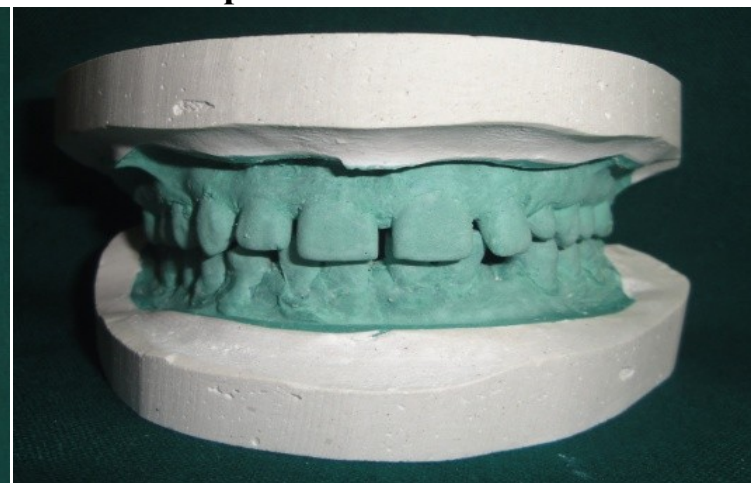

Fig: 4 Hand articulated casts 


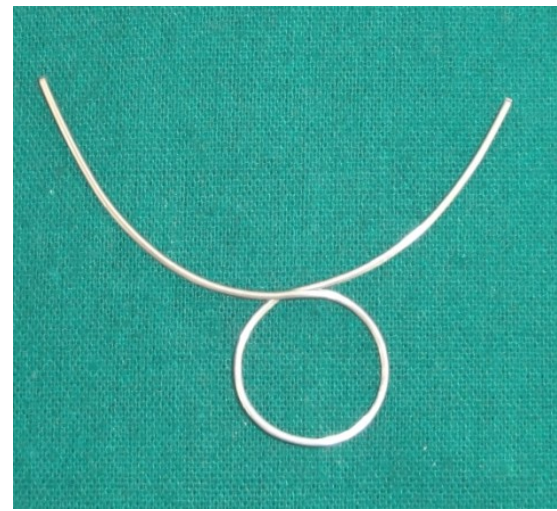

Fig: 5 Wire with Coil in center

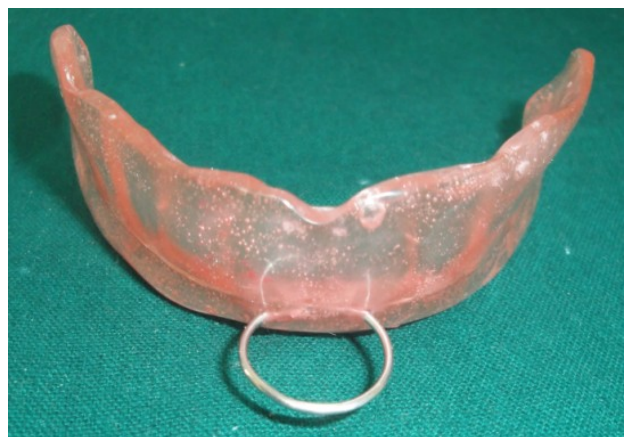

Fig: 6 Completed oral screen prosthesis

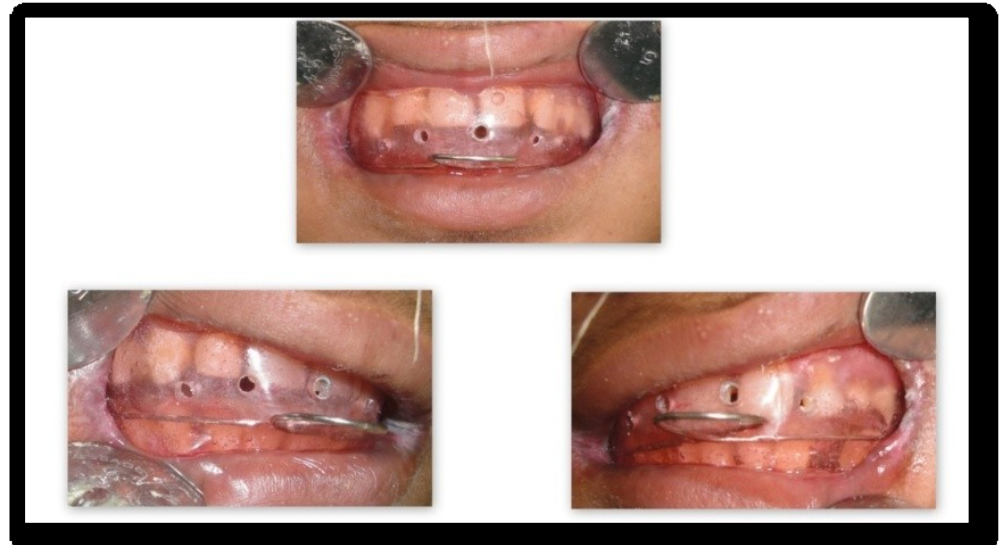

Fig 7: Oral screen appliance inserted in patient's mouth 